\title{
Avaliação da efetividade de participações em programas de ensaio de proficiência no controle da qualidade dos resultados analíticos na determinação de metais e semimetais em águas em laboratório de Saúde Pública
}

\author{
Evaluation of the effectiveness of participation in Proficiency \\ Testing Programs in the quality control of the analytical \\ results in the determination of metals and semimetals \\ in water in a Public Health laboratory
}

Recebido em: 03/05/2019

Aceito em: 20/08/2019

Luciana Juncioni de ARAUZ; Márcia Liane BUZZO;

Lidiane Raquel Verola MATAVELI; Paulo TIGLEA

Núcleo de Contaminantes Inorgânicos, Centro de Contaminantes, Instituto Adolfo Lutz. Av. Dr. Arnaldo, 355, Pacaembú, CEP 01246-000. São Paulo, SP, Brasil.

E-mail:lucianaja2@gmail.com

\section{ABSTRACT}

Proficiency Testing Programs (PTP) have as purpose to evaluate the performance of a laboratory against preestablished criteria using interlaboratory comparisons, as an external evaluation tool in the demonstration of the laboratory analytical reliability, besides being one of the requirements for the accreditation process. For analytical laboratories that work in the quality control of products and services intended for human consumption, obtaining reliable results is fundamental for its credibility and quality assurance. Results issued by the laboratories are evaluated by the Proficiency Testing providers using statistical approaches (z score, zeta score, normalized error, among others) to evaluate the effectiveness of their performances. The goal of this work was to evaluate the effectiveness of the participation of the Inorganic Contaminants Laboratory (ICL), that belongs to the Contaminants Division of the Adolfo Lutz Institute, in different PTP for metals and semimetals in water, from 2013 to 2018. The obtained results demonstrated the effectiveness of the continuous improvement in the execution of the analyzes performed by the ICL, and consequently the reliability of the analytical results. This work aims to disseminate, encourage and provide tools to analytical laboratories that act in the quality control of products and services destined to Public Health, to assist the implementation of corrective and preventive measures, allowing its continuous analytical improvement.

Keywords: metals; water; quality control; chemical analysis; Proficiency Testing Program

\section{RESUMO}

Programas de Ensaios de Proficiência (PEP) têm por finalidade a avaliação do desempenho de um laboratório, contra critérios preestabelecidos por meio de comparações interlaboratoriais, que são utilizados como ferramenta de avaliação externa na demonstração da confiabilidade analítica laboratorial, além de ser um dos requisitos para o processo de acreditação. Para laboratórios analíticos que atuam no controle da quali- 
dade de produtos e serviços destinados ao consumo humano, a obtenção de resultados confiáveis é fundamental para a credibilidade e para a garantia da qualidade das suas funções. Os resultados emitidos pelos laboratórios são avaliados pelos Provedores de Ensaios de Proficiência por meio de métodos estatísticos (índice z, índice zeta, erro normalizado, entre outros) para verificar a eficácia de seus desempenhos. Este trabalho teve como meta avaliar a efetividade da participação do Núcleo de Contaminantes Inorgânicos (NCI) do Centro de Contaminantes do Instituto Adolfo Lutz em diferentes PEPs para metais e semimetais na matriz água, no período compreendido entre 2013 e 2018. Os resultados apresentados demonstraram a efetividade da melhoria contínua realizada na execução das análises pelo NCI e consequentemente o aumento da confiabilidade dos resultados analíticos. Este trabalho visa divulgar, incentivar e fornecer ferramentas aos laboratórios analíticos que atuam no controle da qualidade de produtos e serviços destinados à saúde pública, para auxiliar a implantação de medidas corretivas e preventivas, permitindo seu aprimoramento analítico contínuo.

Palavras-chave: metais; água; controle de qualidade; análises químicas; Programa de Ensaio de Proficiência

\section{INTRODUÇÃO}

Programas de Ensaios de Proficiência (PEP) têm por finalidade a avaliação de desempenho de laboratórios de ensaio ou de calibração acreditados ou postulantes à acreditação, contra critérios preestabelecidos por meio de comparações interlaboratoriais, e são utilizados como ferramenta de avaliação externa na demonstração da competência e da confiabilidade analítica laboratorial, em conformidade com os requisitos de qualidade da Norma ABNT NBR ISO/IEC 17025 (1,2).

Além da obediência à norma, a participação em Programas de Ensaios de Proficiência permite a auto-avaliação de desempenho do laboratório e do analista, complementa a validação da metodologia analítica utilizada, possibilita a comparação de desempenho entre laboratórios, identifica erros analíticos (tendências), permite a tomada de ações corretivas, evidencia a necessidade de treinamentos e mudanças nos procedimentos analíticos e aumenta a confiança por parte dos clientes (3-7).

Assim, o Instituto Adolfo Lutz (IAL), com atuação em pesquisa, inovação tecnológica e ações laboratoriais de Vigilância em Saúde, integrante da rede de Laboratórios Centrais de Saúde Pública do País, vem participando de diversos Programas de Ensaios de Proficiência em distintas áreas de atuação, visando a promoção da confiança na operação de seus laboratórios, bem como na emissão de resultados válidos.
Dentre os laboratórios de ensaios atuantes do IAL, o Núcleo de Contaminantes Inorgânicos (NCI) atende a demandas de análises dos sistemas de vigilância em saúde na determinação de contaminantes inorgânicos (metais e semimetais) em diversos produtos e fluidos biológicos, incluindo a matriz água. Participa de programas de monitoramento de interesse em saúde pública no Estado de São Paulo, desenvolvidos em conjunto com o Centro de Vigilância Sanitária, como o Programa de Vigilância da Água para Consumo Humano (PROÁGUA), o Programa de Monitoramento da Água Tratada para Diálise e Programa Paulista de Análise de Alimentos (água mineral), na avaliação da qualidade dos distintos tipos de água quanto aos níveis de metais e semimetais.

Com o objetivo de garantir a qualidade e a confiabilidade dos resultados emitidos e que são utilizados pelas autoridades sanitárias nas tomadas de ações, visando à promoção da saúde da população, o NCI, entre outras atividades, monitora desde 2002 o seu desempenho nos ensaios de determinação de contaminantes inorgânicos em águas por meio de participação periódica em PEP.

Em 2013, o Núcleo foi acreditado pela Coordenação Geral de Acreditação (CGCRE) do Instituto Nacional de Metrologia, Qualidade e Tecnologia (INMETRO) segundo a Norma ABNT NBR ISO/ IEC 17.025 (1), para ensaios de determinação de metais e semimetais em água tratada para consumo humano, para diálise e envasada (8). 
Diante do exposto, o presente trabalho tem como objetivo avaliar a efetividade das participações do NCI em diversos Programas de Ensaio de Proficiência nacionais para metais e semimetais em água, como instrumento de controle externo da qualidade para assegurar a confiabilidade metrológica das medições analíticas realizadas no laboratório, incluindo a tomada de ações corretivas e preventivas efetuadas em decorrência dessas participações, ao longo de um período de seis anos após o recebimento da acreditação.

\section{MATERIAL E MÉTODO}

\section{Descontaminação do material de laborató-}

rio. Todo o material utilizado nas análises laboratoriais foi previamente imerso em solução de detergente Extran ${ }^{\circledR}$ (Merck) e enxaguados em água corrente. A seguir, foram descontaminados quimicamente por imersão em solução de ácido nítrico $\left(\mathrm{HNO}_{3}\right.$, P.A., Merck) e enxaguados com água ultrapura antes de sua utilização.

Reagentes e soluções analíticas. A água ultrapura utilizada (resistividade 18,2 M $\Omega$.cm) foi obtida a partir de um sistema de purificação de água Millipore (Bedford, EUA) e empregada no preparo das soluções utilizadas.

As soluções padrão monoelementares de Ag, $\mathrm{Al}, \mathrm{As}, \mathrm{Ba}, \mathrm{Be}, \mathrm{Ca}, \mathrm{Cd}, \mathrm{Cr}, \mathrm{Cu}, \mathrm{Ge}, \mathrm{Hg}, \mathrm{In}, \mathrm{K}$, $\mathrm{Mg}, \mathrm{Mn}, \mathrm{Na}, \mathrm{Ni}, \mathrm{Pb}, \mathrm{Re}, \mathrm{Sb}, \mathrm{Sc}, \mathrm{Se}, \mathrm{Tl}$ e Zn foram adquiridas de produtores acreditados de acordo com os requisitos da norma ABNT NBR ISO 17034:2017 (9). Ácido nítrico Suprapur ${ }^{\circledR}$ (Merck, Darmstadt) foi utilizado para o preparo das curvas analíticas e também na solução de padrão interno, para todos os analitos, exceto para mercúrio. Ácido clorídrico (P.A., Merck) foi empregado no preparo da curva analítica de mercúrio.

Os Materiais de Referência Certificados adquiridos do National Institute of Standards Technology (NIST) SRM 1643F e SRM 1640a foram empregados para verificar a exatidão da metodologia analítica para os metais e semimetais. Para o mercúrio, foi utilizada solução padrão com concentração de $1000 \mathrm{mg} / \mathrm{L}$, também adquirida de produtor acreditado pela norma ABNT NBR ISO 17034:2017 (9).

A calibração externa para os elementos em estudo foi realizada utilizando curva analítica preparada em meio ácido. As faixas analíticas utilizadas estão indicadas na Tabela 1 .

Instrumentação. Todas as medidas de determinação dos metais e semimetais (exceto mercúrio) foram realizadas utilizando-se Espectrômetro de Massas com Plasma Indutivamente Acoplado/ ICP-MS (Elan DRC II, Perkin Elmer). As condições operacionais relevantes são mostradas na Tabela 2 .

O nível de mercúrio nas amostras foi determinado pela técnica de Espectrometria de Absorção Atômica com Gerador de Vapor Frio/ASSGV (Analyst 100/FIAS 400, Perkin Elmer) em comprimento de onda de 253,7 nm, utilizando-se lâmpada específica de descarga sem eletrodo (EDL) e sistema amalgamador.

Tabela 1. Faixa de concentração da curva analítica para os analitos em estudo

\begin{tabular}{|c|c|}
\hline Analito & concentração (mg/L) \\
\hline $\mathrm{Be}$ & $0,0004-0,02$ \\
\hline $\mathrm{Na}, \mathrm{Mg}, \mathrm{K}, \mathrm{Ca}$ & $0,1-4,0$ \\
\hline $\mathrm{Ba}, \mathrm{Cu}, \mathrm{Mn}$ & $0,025-1,0$ \\
\hline $\mathrm{Al}, \mathrm{As}, \mathrm{Cr}, \mathrm{Ni}, \mathrm{Pb}, \mathrm{Se}, \mathrm{Tl}, \mathrm{Zn}$ & $0,001-0,05$ \\
\hline $\mathrm{Cd}$ & $0,0005-0,025$ \\
\hline $\mathrm{Ag}, \mathrm{Sb}$ & $0,001-0,05$ \\
\hline $\mathrm{Hg}$ & $0,1-2,0$ \\
\hline $\mathrm{In}, \mathrm{Re}, \mathrm{Sc}, \mathrm{Ge}^{*}$ & 0,005 \\
\hline
\end{tabular}

* Solução de padrão interno

Procedimentos analíticos. As metodologias analíticas validadas atenderam aos valores máximos permitidos para as legislações de águas sujeitas ao regime de vigilância: Portaria de consolidação $\mathrm{n}^{\circ}$ 5/2017 (água para consumo humano) (10), Resolução da Diretoria Colegiada (RDC) no 274/2005 (águas envasadas e gelo) (11) e Resolução RDC n ${ }^{\circ}$ 11/2014 (água tratada para diálise) (12). 
Tabela 2. Condições relevantes de operação do Espectrômetro de Massas com Plasma Indutivamente Acoplado (ICP-MS)

\begin{tabular}{|l|r|}
\hline Potência RF, W & 1450 \\
\hline Fluxo de gás do plasma, L/min & 18 \\
\hline Fluxo do gás auxiliar, L/min & 1,1 \\
\hline Fluxo do gás nebulizador, L/min & 1,02 \\
\hline Tempo de integração, ms & 750 \\
\hline
\end{tabular}

\section{RESULTADOS E DISCUSSÃO}

Diferentes Provedores de Ensaios de Proficiência utilizam distintos modelos estatísticos e formas de apresentação de resultados para as avaliações de desempenho dos participantes, incluindo índice z, erro normalizado, elipse de confiança, índice z' e índice zeta, seguindo critérios de aceitação de resultados estabelecidos pela norma ISO 13.528 (13).

$\mathrm{O}$ índice z e o índice z' representam o afastamento do resultado apresentado pelo participante em relação ao valor designado do PEP, em números de desvios padrão, e o desempenho dos laboratórios é avaliado de acordo com o seguinte critério: $|\mathrm{z}| \leq$ 2 indica desempenho satisfatório; para $2<|\mathrm{z}|<3$, o desempenho é considerado questionável; e para $\mathrm{z} \mid \geq 3$ o desempenho é considerado insatisfatórioO índice zeta considera em seu cálculo as incertezas padrão do valor apresentado pelo participante e do valor designado, sendo a sua avaliação semelhante ao índice $\mathrm{z}$.

Para a aplicação do erro normalizado (En), os laboratórios participantes devem relatar ao provedor também as incertezas expandidas associadas aos seus resultados. Em relação a este critério, quando $\mid$ En $\mid \leq 1$, o resultado é considerado satisfatório, e para $|\mathrm{En}|>1$, insatisfatório.

A avaliação gráfica obtida pelo emprego da elipse de confiança é utilizada para verificar a compatibilidade de resultados entre os laboratórios, por meio da distribuição pelo provedor de pares de amostras de concentrações semelhantes. O gráfico da elipse permite ao participante avaliar seu desempenho com relação aos demais, pela visualização do ponto representativo de seu laboratório, obter informações quanto a erros aleatórios e sistemáticos em seu desempenho analítico, e orientações quanto a possíveis ações corretivas a serem tomadas

A Tabela 3 exibe os resultados obtidos pelo NCI em oito rodadas de PEP promovidos por diferentes provedores. Os resultados consideram os parâmetros das avaliações estatísticas específicas preestabelecidas por cada provedor (índice $z$, erro normalizado, elipse de confiança, índice z' e índice zeta), contidos nos relatórios das rodadas. A tabela mostra ainda o desempenho final do NCI para cada analito, atribuído pelos provedores como satisfatório, insatisfatório ou questionável. 
Tabela 3. Avaliação dos resultados obtidos pelo Núcleo de Contaminantes Inorgânicos em participações em distintos Programas de Ensaio de Proficiência (PEP), nas determinações de metais e semimetais na matriz água, de acordo com a estatística adotada.

\begin{tabular}{|c|c|c|c|c|c|c|c|}
\hline \multicolumn{2}{|c|}{ Analito } & İndice $z$ & Erro normalizado & $\begin{array}{l}\text { Elipse de } \\
\text { confiança }\end{array}$ & Índice $z$ & $\begin{array}{c}\text { Indice } \\
\text { Zeta }(\zeta)\end{array}$ & Desempenho \\
\hline \multicolumn{8}{|c|}{ SENAI/CETIND Rodada 005/2013 } \\
\hline Al & $A / B$ & $0,32 / 0,72$ & $2,91 / 2,24$ & Satisfatório & - & - & Satisfatório \\
\hline $\mathrm{Ba}$ & $A / B$ & $-0,18 /-0,29$ & $0,57 /-0,15$ & Satisfatório & - & - & Satisfatório \\
\hline Cd & $A / B$ & $1,19 / 0,71$ & $0,69 / 0,16$ & Satisfatório & - & - & Satisfatório \\
\hline $\mathrm{Ca}$ & $A / B$ & $0,91 / 1,24$ & $0,53 / 0,91$ & Satisfatório & - & - & Satisfatório \\
\hline $\mathrm{Pb}$ & $A / B$ & $-0,10 /-0,15$ & $0,07 /-0,02$ & Satisfatório & - & - & Satisfatório \\
\hline $\mathrm{Cu}$ & $A / B$ & $-2,44 / 1,40$ & $-2,61 / 0,69$ & Insatisfatório & - & - & Insatisfatório \\
\hline $\mathrm{Cr}$ & $A / B$ & $-1,62 /-0,28$ & $0,06 / 0,13$ & Satisfatório & - & - & Satisfatório \\
\hline $\mathrm{Mg}$ & $A / B$ & $-0,30 / 0,58$ & $-0,08 / 0,66$ & Satisfatório & - & - & Satisfatório \\
\hline $\mathrm{Mn}$ & $A / B$ & $-0,16 /-1,56$ & $0,61 /-1,17$ & Satisfatório & - & - & Satisfatório \\
\hline $\mathrm{Ni}$ & $A / B$ & $-0,11 / 0,40$ & $0,09 / 1,29$ & Satisfatório & - & - & Satisfatório \\
\hline As & $A / B$ & $-0,23 /-0,16$ & $0,69 / 1,13$ & Satisfatório & - & - & Satisfatório \\
\hline Se & $A / B$ & $-0,06 / 0,07$ & $1,12 / 0,79$ & Satisfatório & - & - & Satisfatório \\
\hline Sb & $A / B$ & $0,37 / 1,10$ & $3,56 / 6,47$ & Satisfatório & - & - & Satisfatório \\
\hline K & $\mathrm{A} / \mathrm{B}$ & $-1,31 /-1,34$ & $-0,17 /-0,05$ & Satisfatório & - & - & Satisfatório \\
\hline $\mathrm{Ag}$ & $A / B$ & $0,15 / 0,49$ & $0,71 / 1,14$ & Satisfatório & - & - & Satisfatório \\
\hline $\mathrm{Na}$ & $A / B$ & $-0,08 /-0,25$ & $0,21 / 0,47$ & Satisfatório & - & - & Satisfatório \\
\hline $\mathrm{Zn}$ & $A / B$ & $-0,26 /-0,23$ & $0,17 / 0,78$ & Satisfatório & - & - & Satisfatório \\
\hline $\mathrm{Hg}$ & $A / B$ & $-0,96 /-0,80$ & $-2,91 /-4,51$ & Satisfatório & - & - & Satisfatório \\
\hline \multicolumn{8}{|c|}{ INMETRO $5^{\text {a }}$ Rodada/2014 } \\
\hline As & & - & $-0,6$ & - & - & - & Satisfatório \\
\hline $\mathrm{Cr}$ & & - & 3,8 & - & - & - & Insatisfatório \\
\hline $\mathrm{Pb}$ & & - & 0,4 & - & - & - & Satisfatório \\
\hline $\mathrm{Mn}$ & & - & 8,5 & - & - & - & Insatisfatório \\
\hline \multicolumn{8}{|c|}{${ }^{1}$ SENAI/CETIND Rodada 001/2014 } \\
\hline $\mathrm{Mn}$ & & - & - & - & - & - & Satisfatório \\
\hline $\mathrm{Na}$ & & - & - & - & - & - & Satisfatório \\
\hline $\mathrm{Zn}$ & & - & - & - & - & - & Satisfatório \\
\hline $\mathrm{Ni}$ & & - & - & - & - & - & Satisfatório \\
\hline $\mathrm{Ba}$ & & - & - & - & - & - & Satisfatório \\
\hline \multicolumn{8}{|c|}{ RMMG 3 ${ }^{\text {a }}$ Rodada AA-03/2015 } \\
\hline $\mathrm{Ba}$ & & 0,0 & - & - & - & - & Satisfatório \\
\hline Cd & & 0,6 & - & - & - & - & Satisfatório \\
\hline $\mathrm{Pb}$ & & 0,6 & - & - & - & - & Satisfatório \\
\hline $\mathrm{Cu}$ & & 0,6 & - & - & - & - & Satisfatório \\
\hline $\mathrm{Cr}$ & & 0,9 & - & - & - & - & Satisfatório \\
\hline $\mathrm{Mn}$ & & 0,8 & - & - & - & - & Satisfatório \\
\hline $\mathrm{Ni}$ & & 0,7 & - & - & - & - & Satisfatório \\
\hline $\mathrm{Zn}$ & & $-1,0$ & - & - & - & - & Satisfatório \\
\hline \multicolumn{8}{|c|}{${ }^{2}$ SENAI Rodada 005/2015 } \\
\hline Al & $A / B$ & $1,13 / 1,22$ & $1,00 / 1,16$ & Satisfatório & - & - & Satisfatório \\
\hline $\mathrm{Cd}$ & $\mathrm{A} / \mathrm{B}$ & $0,55 / 0,34$ & $2,66 / 3,74$ & Satisfatório & - & - & Satisfatório \\
\hline $\mathrm{Ca}$ & $\mathrm{A} / \mathrm{B}$ & $0,89 / 0,32$ & $1,08 / 0,39$ & Satisfatório & - & - & Satisfatório \\
\hline $\mathrm{Pb}$ & $\mathrm{A} / \mathrm{B}$ & $0,62 / 0,65$ & $0,86 / 0,94$ & Satisfatório & - & - & Satisfatório \\
\hline $\mathrm{Cu}$ & $\mathrm{A} / \mathrm{B}$ & $0,29 / 0,12$ & $2,11 / 0,74$ & Satisfatório & - & - & Satisfatório \\
\hline
\end{tabular}


Tabela 3 (Cont.). Avaliação dos resultados obtidos pelo Núcleo de Contaminantes Inorgânicos em participações em distintos Programas de Ensaio de Proficiência (PEP), nas determinações de metais e semimetais na matriz água, de acordo com a estatística adotada.

\begin{tabular}{|c|c|c|c|c|c|c|c|}
\hline \multicolumn{8}{|c|}{${ }^{2}$ SENAI Rodada 005/2015 } \\
\hline $\mathrm{Cr}$ & $A / B$ & $0,07 /-0,04$ & $0,08 /-0,05$ & Satisfatório & - & - & Satisfatório \\
\hline $\mathrm{Mg}$ & $A / B$ & $0,80 / 1,17$ & $1,38 / 1,87$ & Satisfatório & - & - & Satisfatório \\
\hline $\mathrm{K}$ & $A / B$ & $0,12 /-0,13$ & $0,49 /-0,56$ & Satisfatório & - & - & Satisfatório \\
\hline $\mathbf{A g}$ & $A / B$ & $0,94 /-0,16$ & $0,71 /-0,16$ & Satisfatório & - & - & Satisfatório \\
\hline $\mathrm{Se}$ & $A / B$ & $-1,13 /-0,95$ & $-3,10 /-0,95$ & Satisfatório & - & - & Satisfatório \\
\hline $\mathrm{Hg}$ & $A / B$ & $0,00 /-0,55$ & $0,01 /-0,93$ & Satisfatório & - & - & Satisfatório \\
\hline Mn & $A / B$ & $0,00 /-0,37$ & $-0,01 /-0,56$ & Satisfatório & - & - & Satisfatório \\
\hline $\mathrm{Na}$ & $A / B$ & $0,72 / 0,87$ & $0,69 / 0,76$ & Satisfatório & - & - & Satisfatório \\
\hline $\mathrm{Zn}$ & $A / B$ & $-1,05 /-0,64$ & $-1,49 /-0,75$ & Satisfatório & - & - & Satisfatório \\
\hline $\mathrm{Ni}$ & $A / B$ & $-0,05 /-0,14$ & $-0,20 /-0,44$ & Satisfatório & - & - & Satisfatório \\
\hline $\mathrm{Ba}$ & $A / B$ & $0,10 /-0,08$ & $0,14 /-0,10$ & Satisfatório & - & - & Satisfatório \\
\hline \multicolumn{8}{|c|}{ INMETRO $7^{\text {a }}$ Rodada/2017 } \\
\hline As & & - & - & - & - & 0,6 & Satisfatório \\
\hline Cd & & - & - & - & - & 0,8 & Satisfatório \\
\hline Mn & & - & - & - & - & $-1,4$ & Satisfatório \\
\hline $\mathrm{Ni}$ & & - & - & - & - & 0,4 & Satisfatório \\
\hline $\mathrm{Pb}$ & & - & - & - & - & $-0,5$ & Satisfatório \\
\hline \multicolumn{8}{|c|}{${ }^{3} \mathrm{TOQ}$ SABESP $1 \mathrm{AP} / 2018$} \\
\hline Sb & & - & - & - & $-0,09$ & - & Satisfatório \\
\hline As & & 0,02 & - & - & - & - & Satisfatório \\
\hline $\mathrm{Ba}$ & & 0,24 & - & - & - & - & Satisfatório \\
\hline Cd & & - & - & - & 0,44 & - & Satisfatório \\
\hline $\mathrm{Pb}$ & & $-0,04$ & - & - & - & - & Satisfatório \\
\hline $\mathrm{Cu}$ & & - & - & - & $-0,05$ & - & Satisfatório \\
\hline $\mathrm{Cr}$ & & - & - & - & $-0,26$ & & Satisfatório \\
\hline $\mathrm{Ni}$ & & - & - & - & $-0,71$ & - & Satisfatório \\
\hline Se & & - & - & - & 0,11 & - & Satisfatório \\
\hline $\mathrm{Zn}$ & & 0,57 & - & - & - & - & Satisfatório \\
\hline \multicolumn{8}{|c|}{ INMETRO $8^{\mathrm{a}}$ Rodada/2018 } \\
\hline As & & - & - & - & $-0,1$ & - & Satisfatório \\
\hline Cd & & - & - & - & $-0,5$ & - & Satisfatório \\
\hline $\mathrm{Na}$ & & $-2,4$ & - & - & - & - & Questionável \\
\hline $\mathrm{Ni}$ & & $-0,1$ & - & - & - & - & Satisfatório \\
\hline $\mathrm{Pb}$ & & 0,7 & - & - & $-0,6$ & - & Satisfatório \\
\hline $\mathrm{Zn}$ & & 0,7 & - & - & - & - & Satisfatório \\
\hline
\end{tabular}

As letras A e B representam o número de amostras distintas e com concentrações semelhantes para os elementos em estudo, recebidas na rodada do PEP.

${ }^{1}$ O NCI foi convidado como laboratório especialista para participar deste PEP, visando compor o valor designado neste Programa. 0 parâmetro de avaliação (índice z) não foi discriminado numericamente no relatório, o qual exibe somente o formato gráfico.

${ }^{2}$ Os resultados da participação do NCI para o elemento arsênio foram enviados ao PEP, porém o provedor cancelou a avaliação estatística para este elemento na rodada porque a amostra não apresentou homogeneidade satisfatória.

${ }^{3} 0$ resultado da participação do NCI para o elemento $\mathrm{Hg}$ foi enviado ao PEP, porém o provedor retirou este elemento da rodada devido a problemas de ordem técnica. 
De modo geral, a análise da Tabela 3 indica que o NCI vem apresentando maiores índices de desempenhos satisfatórios para os analitos em estudo. Este fato é melhor evidenciado ao considerar o conjunto de 72 ensaios efetuados ao longo do intervalo de estudo, onde a atribuição de desempenho satisfatório para cada analito emitida pelos PEPs corresponde a $94 \%$ do total, o que aponta para a efetividade da melhoria contínua da qualidade implantada na execução de análises laboratoriais, com aumento da confiabilidade do resultado emitido.

Os valores obtidos pelo NCI quanto ao índice $\mathrm{z}$, índice z' e índice zeta em suas participações nos diferentes PEPs, mostraram-se dentro do intervalo de aceitação descrito pela norma ISO 13.528 (13), indicando que o laboratório possui procedimentos de controle da qualidade implantados de forma adequada, a fim de assegurar a validade dos ensaios realizados.

Por outro lado, somente $6 \%$ do total de elementos analisados estavam em desacordo com a ferramenta estatística adotada no respectivo PEP. As exceções foram constatadas para os analitos cobre pertinente à participação na rodada SENAI/CETIND Rodada 005/2013; cromo e manganês pertinente à rodada INMETRO $5^{\mathrm{a}}$ Rodada/2014, e sódio pertinente à rodada INMETRO $8^{\mathrm{a}}$ Rodada/2018; em que os Provedores atribuíram desempenhos insatisfatório ou então questionável para estes metais.

Os resultados obtidos em atividades de ensaios de proficiência classificados como insatisfatório ou questionável, de acordo com a metodologia estatística adotada pelos PEP, e ainda de acordo com os requisitos de qualidade da norma ABNT NBR ISO/ IEC 17.025 (1), indicam a necessidade de investigação da causa raiz, avaliação da não conformidade gerada, tomada de ação corretiva ou preventiva pertinente, verificação da eficácia das ações implementadas, incluindo posterior participação em nova rodada de ensaio de proficiência $(1,2,14,15)$.

O erro normalizado (En) aplicado como uma ferramenta de avaliação estatística, não indica, necessariamente, a proximidade do resultado do laboratório em relação ao valor designado, não sendo o critério principal para a classificação do laboratório em relação a esse parâmetro, quando outro indicador, como o índice $\mathrm{z}$, for utilizado. Um alto valor de En pode indicar que o resultado esteja afastado do valor referência, mas também pode refletir uma estimativa subestimada da incerteza. Dessa forma, há participações em que, apesar de o laboratório ter apresentado um erro normalizado fora da faixa de aceitação, obteve o desempenho avaliado como satisfatório segundo o índice z. No caso em que o valor de erro normalizado foi aplicado como critério principal, na avaliação estatística do INMETRO $5^{\mathrm{a}}$ Rodada/2014, os elementos cromo e manganês não atenderam ao critério de aceitação previsto pela norma ISO 13.528 (12). Ainda, dentre todos os ensaios executados somente para o cobre na rodada referente ao SENAI/CETIND Rodada 005/2013, o diagrama de dispersão da elipse de confiança demonstrou resultado insatisfatório.

Assim, nos casos citados acima, a fim de garantir resultados precisos e confiáveis, o laboratório vem implantando ações corretivas e preventivas quando desempenhos insatisfatório ou questionável são observados, visando à garantia de qualidade por meio do sistema de gestão, que engloba: a melhoria contínua da documentação (procedimentos operacionais padrão); participações periódicas em auditorias internas e reavaliações externas efetuadas pela CGCRE/INMETRO e implementação constante do controle interno da qualidade.

Dentre as outras principais abordagens efetivadas no controle interno da qualidade laboratorial, ao longo do período de estudo, destacaram-se:

a) Emprego rotineiro nas análises de emprego de Material de Referência Certificado (MRC) obtido de fornecedor acreditado de acordo com a norma ABNT NBR ISO 17.034 quando pertinente ou, na ausência deste, o uso de Material de Referência (MR) ou ainda a utilização de adição de padrão, em todas as tomadas de ensaios, com base em critérios de aceitação preestabelecidos.

b) Implantação da utilização de cartas de controle no monitoramento de processos de medição quantitativos para o uso de MRC, MR e adição de padrão, bem como para o branco da análise em todas as tomadas de ensaios, com base em critérios de aceitação preestabelecidos; 
c) Aprimoramento da estimativa do cálculo de incerteza para os analitos, com a identificação e aplicação de todas as fontes de incerteza previstas e suas respectivas contribuições, como: influência dos instrumentos de medição (calibração e resolução), pureza dos padrões, incerteza da concentração dos materiais de referência, influência das condições ambientais (temperatura, umidade), além da regressão da curva analítica com ajuste linear e precisão (repetibilidade, precisão intermediária e reprodutibilidade);

d) Participação em novo ensaio de proficiência, como objeto de avaliação final da eficácia das resoluções das não conformidades geradas, a fim de garantir continuamente a qualidade dos resultados analíticos emitidos.

Assim, com intuito de melhorar a competividade, bem como a emissão de resultados confiáveis dos laboratórios participantes a seus clientes, a literatura recente disponibiliza importantes avaliações críticas de Provedores de Ensaios de Proficiência em suas áreas de atuação, onde são apresentadas as evidências de progresso, bem como são abordadas as dificuldades na implantação e manutenção das rodadas (16-29).

Dentre estes diagnósticos, as pesquisas apontam para a necessidade de verificação de desvio do desempenho quando resultados insatisfatórios ou questionáveis são obtidos pelos participantes, na análise de causa raiz e consequente implementação de ações corretivas ou preventivas, seguida de verificação de eficácia de tomada de ação.

Apesar de tratar-se de objeto distinto do presente estudo, onde é demonstrada a autoavaliação e a evolução em diversas participações de um único laboratório em distintos PEP, estas recomendações foram primordiais para aplicação no NCI, e vêm corroborando na emissão de resultados confiáveis no seguimento dos requisitos implantados pelo sistema de gestão de qualidade, com vistas ao atendimento da norma ABNT ISO/IEC NBR 17.025 (1).

\section{CONCLUSÃO}

Os resultados apresentados visam divulgar, incentivar e fornecer ferramentas aos laboratórios analíticos que atuam no controle de produtos e serviços destinados ao consumo humano, na obtenção de resultados com exatidão e precisão requeridas, imprescindíveis para a credibilidade do laboratório e para sua garantia da qualidade. Ressalta-se que a participação em programas interlaboratoriais pode identificar erros que, muitas vezes, não ficam evidentes na rotina das análises de um laboratório, e que podem afetar decisões econômicas e políticas, causando prejuízo ao meio ambiente e à saúde da população.

Estes fatos denotam, ainda, a importância da efetivação da autoavaliação na participação constante de laboratórios analíticos, acreditados ou postulantes à acreditação, em ensaios de proficiência utilizados como controle externo da qualidade, visando à melhoria contínua de emissão de resultados confiáveis aos clientes como demonstração de competência técnica.

\section{AGRADECIMENTOS}

A Edna E. K. Arakaki pela realização das análises de mercúrio total e Camila C. de Oliveira pelo auxílio na revisão do texto. 
1. ABNT. Associação Brasileira de Normas Técnicas. Norma ABNT NBR ISO/IEC 17025:2017. Requisitos gerais para competência de laboratórios de ensaio e calibração. Rio de Janeiro, 2017.

2. INMETRO. Instituto Nacional de Metrologia, Qualidade e Tecnologia. Requisitos para a participação de laboratórios em atividades de ensaio de proficiência. Norma NIT-DICLA-026, Revisão Nº 11. P. 13. Acesso em: [25 fev 2019]. Disponível em:http://www.inmetro.gov.br/Sidoq/ pesquisa_link.asp?seq_tipo_documento $=4 \&$ cod_uo_numeracao $=00587$ \&num_documento $=026$.

3. Charki A, Pavese F. Data comparisons and uncertainty: a roadmap for gaining in competence and improving the reliability of results. Int J Metrol Qual Eng. 2019;10(1):110. DOI: $10.1051 /$ ijmqe/2018016

4. Gumma V, Kyle DeGruy K, Bennett D, Kim TNT, Albert H, Bond KB, Gutreuter S, Alexander H, Ngyuen Thi Phong L, Rush TH, et al. Impact of external quality assurance on the quality of Xpert MTB/RIF testing in Viet Nam. Impact of external quality assurance on the quality of Xpert MTB/RIF testing in Viet Nam. J Clin Microbiol. 2019;57:e01669-18. DOI: 10 .1128/JCM.01669-18

5. Gajadhar AA, Noeckler K, Boireau P, Rossi Pascal, Scandrett B, Gamble HR. International commission on Trichinellosis: Recommendations for quality assurance in digestion testing programs for Trichinella. Food Waterborne Parasitol. 2019; e00059. DOI: .1016/j.fawpar.2019.e00059

6. Caldwell KL, Cheng P-Y, Vance KA, Makhmudov A, Jarrett JM, Caudill SP, Ho DP, Jones RL. LAMP: A CDC Program to Ensure the Quality of Blood-Lead Laboratory Measurements. J Public Health Manag Pract. 2019;25(1 Supp), S23-S30. DOI: 10.1097/PHH.0000000000000886

7. Ferrini AM, Appicciafuoco B, Massaro MR, Galati G, Patriarca M. Proficiency testing as an instrument to assess the analytical performance and the methods routinely implemented: the italian experience for the screening of antibiotic residues in milk in the official control. Accreditation and Quality Assurance. 2019;24:85-91. DOI: 10.1007/s00769-018-1352-6

8. INMETRO. Instituto Nacional de Metrologia, Qualidade e Tecnologia. Informações sobre o escopo da acreditação CRL 0679. Acesso em: [14 jun 2019]. Disponível em: [http://www. inmetro.gov.br/laboratorios/rble/docs/ CRL0679.pdf].

9. ABNT. Associação Brasileira de Normas Técnicas. Norma ABNT NBR ISO/IEC 17034:2017. Requisitos gerais para a competência de produtores de material de referência. Rio de Janeiro, 2017.

10. BRASIL. Ministério da Saúde. Portaria de Consolidação MS n 5, de 28 de setembro de 2017. Dispõe sobre os procedimentos de controle e de vigilância da qualidade da água para consumo humano e seu padrão de potabilidade. Diário Oficial da União, n 190,03 de outubro de 2017. Seção 1. p 360.

11. BRASIL. Agência Nacional de Vigilância Sanitária. Resolução RDC n 274, de 22 de setembro de 2005. Aprova o Regulamento Técnico para águas envasadas e gelo. Diário Oficial da União, n⿳ 184, 23 de setembro de 2005. Seção I. p 376.

12. BRASIL. Agência Nacional de Vigilância Sanitária. Resolução RDC n ${ }^{\circ} 11$, de 13 de janeiro de 2014. Dispõe sobe os Requisitos de Boas Práticas de Funcionamento para os Serviços de Diálise e dá outras providências. Diário Oficial da União, $n^{\circ}$ 50, 14 de março de 2014, Seção 1. p 40-42.

13. ISO. International Organization for Standardization. International Standard ISO 13.528:2015. Statistical methods for use in proficiency testing by interlaboratory comparison. $2^{\mathrm{a}}$ ed. 2015. Acesso em: [25 fev 2019]. Disponível em: https://www.sis.se/api/docuent/preview/919164/

14. Pizzolato M, Nagel FB, Albano FM. An analysis of ISO/ IEC 17025 nonconformities reported for audits in Brazil. NCSLI Measure J Meas Sci. 2015;10(2):66-74. DOI: 10.1080/19315775.2015.11721727

15. Silva MAF, Moura MH, Nogueira R, Costa SRR. Cause analysis for unsatisfactory results in proficiency testing activities: a case study of Brazilian calibration laboratories accredited under ISO/IEC 17025:2005. Int J Metrol Qual Eng. 2013;4:87-95. DOI: 10.1051/ijmqe/2013041

16. Dajay LC, Portugal TR, Climaco JC, Parcon MRV, Udarbe MA, Placio REE, Adona Jr PE. Establishment of proficiency testing programs in the Philippines. Accred Quality Assur. 2019;24:65-71. DOI: 10.1007/s00769018-1363-3

17. Zamengo L, Tedeschi G, Frison G, Griffoni C, Ponzin D, Jones AW. Inter-laboratory proficiency results of blood alcohol determinations at clinical and forensic laboratories in Italy. Forensic Sci Int. 2019;295:213-218. DOI: 10.1016/j.forsciint.2018.12.018

18. Thiex N, Carlson M, Kiefer R, Kiefer A, Eisenberg D, Barashkov N, Ramsey C. Evaluation of the use of Microtracers $^{\mathrm{TM}}$ in a Proficiency Testing Program. J AOAC Int. 2019;102(3):767-775. DOI: /10.5740/jaoacint.18-0354

19. Rodrigues Filho BA, Farias RF, Anjos W. Implementing a proficiency test provider for sphygmomanometers in Brazil. J Physics: Conf Series. 2018;1044:012033. DOI: 10.1088/1742-6596/1044/1/012033

20. Cao ZT, Botelho JC, Rej R, Vesper H, Astles JR. Impact of testosterone assay standardization efforts assessed via accuracy based proficiency testing. Clin Bioch. 2019;68:3743. DOI: 10.1016/j.clinbiochem.2019.03.014 
21. Getachew AK, Cheneke W, Asres Y, Bekele S, Kebede E. Assessment of coverage and quality of selected clinical chemistry tests among medical laboratories of health facilities in Jimma Zone, South West Ethiopia. J Trop Med. 2019; ID 5954313;1-8. DOI: 10.1155/2019/5954313

22. Derra FA, Mariam BH, Biza T, Legesse T, Muzeyin R, Girma S, Beyene Y, Gonfa A, Ayana G, Lemma E. Assessment of external quality assurance scheme participation level, on Salmonella and Shigella species. Open Microbiol J. 2019;13:55-62. DOI: 10.2174/1874285801913010055

23. Generali T, Stefanelli P, Girolimetti S, Barbini DA. Results of the 16th proficiency test on the determination of pesticide residues in olive oil. Accred Quality Assur 2019;24(1):13-18. DOI: 10.1007/s00769-018-1329-5

24. Mazzoni C, Boubetra A, Nguyen S, Tirard A. Microbiology proficiency testing schemes in wine. Accred Quality Assur. 2019;24:43-48. DOI: 10.1007/s00769-018-1342-8

25. Krismastuti FSH, Aryana N, Hindayani A, Hamim N. Proficiency testing for $\mathrm{pH}$ measurements: a 3year evaluation. Accred Quality Assur. 2019;24:57-63. DOI: 10.1007/s00769-018-1362-4
26. Benilda S. Ebarvia BS, Dacuya A, Cabanilla SR, Mamplata NR. Provision of proficiency testing for histamine mass fraction in canned tuna to improve the capability of chemical laboratories in the Philippines Accred Quality Assur. 2019;24:79-84. DOI: 10.1007/s00769-018-1347-3

27. Kim H, Hwang E, Park J, Heo SW, Yim Y-H, Lim Y, Lim MC, Lee JW, Lee K-S. Proficiency testing for total mercury in oyster with a metrologically traceable reference value from isotope dilution mass spectrometry: implications on laboratory practices using mercury analyzers. Accred Quality Assur. 2019;24(4):253-261. DOI: 10.1007/s00769-019-01379-7

28. Ferrer C, Lozano A, Samanta Uclés S, Valverde A, Amadeo R. Fernández-Alba AR. European Union proficiency tests for pesticide residues in fruit and vegetables from 2009 to 2016: Overview of the results and main achievements. Food Control. 2017;82:101-113. DOI: 10.1016/j. foodcont.2017.06.020

29. Helmersson-Karlqvist J, Ridefelt P, Boija EE, Nordin $\mathrm{G}$. Lower creatinine concentration values and lower inter-laboratory variation among Swedish hospital laboratories in 2014 compared to 1996: results from the equalis external quality assessment program. Clin Chem Lab Med. 2019;57(6):838-844. DOI: 10.1515/cclm-2018-0670 